\title{
Terminalia Catappa Extract (TCE) Reduces Proliferation of Lung and Breast Cancer Cell by Modulating miR-21 and miR-34a Expressions
}

\author{
Habib Zarredar ${ }^{1}$, Amir Mahdi Khamaneh ${ }^{2}$, Fatemeh Firouzi Amoodizaj ${ }^{3}$, \\ Dariush Shanehbandi ${ }^{2}$, Ensiyeh Seyedrezazadeh ${ }^{1}$, Hamed Sabagh Jadid ${ }^{1}$, Milad \\ Asadi $^{4,5}$, Venus Zafari ${ }^{1}$, Yeganeh Khalili ${ }^{2}$, Zahra Soleimani ${ }^{1}$, Atefeh Ansarin ${ }^{1}$, \\ Majid Khalilii ${ }^{2 *}$
}

\begin{abstract}
Background: After cardiovascular illness, cancer is the one of the main and second cause of death in the worldwide. Despite significant advances in this field, low survival, drug resistance, and side effects of chemotherapy remain an unsolved problem. Due to the high mortality rate among cancer patients, finding the new substance to treatment with low side effects is important. Previous studies have been informed that positive effects of herbal medicines on cancer patients, which are very efficient in the treatment of cancer. Methods: In this study, the antitumor effect of ethanolic Terminalia catappa leaf extract (TCE) on MCF-7, MDA-231, and A549 cell lines was examined. For this reason, the effects of TCE on cell migration, gene expression, and growth were investigated by scratch, test, real-time PCR (qPCR) qPCR, and MTT tests respectively. Results: As a reported by the MTT outcomes, TCE significantly decreased the viability of A549, MCF-7, and MDA-231 cells $(\mathrm{P}<0.05)$. Moreover, genes expression patterns that are related to proliferation (miR-21, miR-34a), migration (MMP-13, Vimentin), and apoptosis (Cas-3, Cas-8, Cas-9, Bcl-2, Bax) also have changed significantly after treatment with TCE. Also, in the A549 cell line, Bax ( $p$ value: 0.029 ), Cas-9 ( $p$ value: 0.00023 ), miR-34a (p value: 0.031), Bcl-2 (p value: 0.0076), MMP-13 (p value: 0.041 ), Cas-3 (p value: 0.00051 ) and in MCF-7 cell line Bax (p value: 0.0004 ), Cas-3 (p value: 0.0003 ), Cas-9(p value: 0.037 ), miR-34a (p value: 0.005 ), Bcl-2(pvalue:0.0007), mir-21 (p value:0.016), MMP-13(p value: 0.011) and in MDA-231 cell line $B a x(p$ value $<0.0001$ ), Cas-3(p value: 0.003 ), Cas-9(p value: 0.0004). mir-34a ( $\mathrm{p}$ value:0.0019), Bcl-2( $\mathrm{p}$ value:0.0023), MMP-13( $\mathrm{p}$ value: 0.032 ) have significantly changed compare to control group. Conclusion: The outcomes of this research determined that T. Catappa might be a potential source of antitumor compounds and could be a candidate for further research.
\end{abstract}

Keywords: Metastasis- Terminalia Catappa- breast cancer- apoptosis- lung cancer

Asian Pac J Cancer Prev, 22 (4), 1157-1163

\section{Introduction}

Cancer is defined as an immoderate and uncontrolled progression of cells. Cancer cells are able to spread around tissues and metastasize to distant organs utilizing lymphatic system or blood vessel (Fidler, 1989; Rusciano, 1992). Mutations and irregular patterns of gene expression lead to abnormal gene function which is the main reason for cancer progression ( $\mathrm{Li}$ et al., 2000). Annually, 10 million people die from cancer in the world (Saika and Sobue, 2013). Even though there is a remarkable improvement in early diagnosis and treatment of tumor, but still rate of mortality is high (Bertucci et al., 2012; Bray et al., 2012).

Accordingly, this study focuses on two main types of cancer, namely breast and lung cancers, which are the most prevalent malignancies in humans. Breast cancer is the most prevalent type of cancer in women, with about half of million deaths and 1.5 million new cases every year (Ferlay et al., 2015). Lung cancer is the main reason of cancer-related deaths with $1,761,007$ deaths $(18.4 \%$ of the total cancer-related deaths) and 2,093,876 newly

\footnotetext{
${ }^{1}$ Tuberculosis and Lung Disease Research Center, Tabriz University of Medical Science, Daneshgah Street, Tabriz, Iran. ${ }^{2}$ Tuberculosis and Lung Disease Research Center \& Rahat Breathe and Sleep Resaerch, Center, Tabriz University of Medical Science, Tabriz, Iran. ${ }^{3}$ Department of Genetics, Tabriz Branch, Islamic Azad University, Tabriz, Iran. ${ }^{4}$ Immunology Research Center, Tabriz University of Medical Sciences, Tabriz, Iran. ${ }^{5}$ Department of Basic Oncology, Institute of Health Sciences, Ege University, Izmir, Turkey.*For Correspondence: khalili876@gmail.com
} 
diagnosed cases in 2018 (Ferlay et al., 2010; Stewart, 2015). Currently, treatment protocol of cancer including surgery, chemotherapy, radiography, and immunotherapy is the basis of cancer treatment. Although, chemotherapy is a more commonly used method, it has several problems including toxicity, drug resistance, and limited effectiveness (Tan et al., 2011).

On the other hand, herbal drugs have been used for centuries to treat and prevent diseases. In this regard, the Terminalia catappa (Combretaceae family) plant that is a native plant of India, has been studied for its antitumor properties (Ezeokonkwo, 2004). The previous studies have shown that T.catappa leaf extract (TCE) has antioxidant (Kinoshita et al., 2007), anti-inflammatory, anti-metastatic ,and anti-tumor effects (Fan et al., 2004; Yang et al., 2010). Also, punicalagin as the main part of TCE, has been found to have useful properties against bleomycin-induced genotoxicity in ovary cells of the Chinese hamster (Chen et al., 2000) and chemopreventive effect on H-ras-transformed NIH3T3 cells (Chen and Li, 2006). Also, TCE has been indicated to have effective antimutagenicity and has been more cytotoxic to human hepatoma cells compared to normal liver cells (Ko et al., 2003).

Therefore, in this study, the apoptotic, anti-cancer, and cytotoxic effects of TCE on A549, MCF7, and MDA231 cell lines are investigated. Also, expression of important genes correlated with metastasis, proliferation, and apoptosis is assessed by quantitative polymerase chain reaction (qPCR).

\section{Materials and Methods}

\section{Preparation of TCE}

In the present study, plant leaves were collected from Rudan City (Hormozgan Province, Iran). Healthy green leaves without pests and diseases were selected for the study. Then, $30 \mathrm{~g}$ of leaves was dried and powdered at 40 ${ }^{\circ} \mathrm{C}$ for $12 \mathrm{~h}$. Then, the powdered leaves were dissolved in $50 \%$ ethanol and were extracted by maceration technique 3 times. In the next step, the vacuum rotary evaporator was used to separate solvent from the extracted compound to obtain TCE. Finally, for using TCE, $5 \mathrm{mg}$ of the extract was solved in dimethyl sulfoxide (DMSO) $(100 \mu \mathrm{l})$ solution and then, was added to complete culture medium $(900 \mu \mathrm{l})$.

\section{Cancer Cell Cultures}

MCF7, MDA 231, and A549 cancer cell lines were obtained from the Pasteur Institute of Iran (Tehran, Iran). The prepared cancer cells were cultured on RPMI1640 medium supplemented with $1 \%$ antibiotics including penicillin $(100 \mathrm{IU} / \mathrm{ml})$ plus streptomycin $(100 \mu \mathrm{g} / \mathrm{ml})$ (SigmaAldrich, USA) and fetal bovine serum (FBS) $10 \%$ (Biochrom, Berlin), under the condition of $5 \% \mathrm{CO}_{2}$ atmosphere, $95 \%$ of humidity, and temperature of $37^{\circ} \mathrm{C}$. All the experiments were done when the cells reached logarithmic phase.

\section{Cytotoxicity Assay}

A number of 104 cancer cells were seeded in 96-well plates. The cells were treated for $24-72 \mathrm{~h}$ at $37^{\circ} \mathrm{C}$ with different concentrations of TCE $(0.5,1,2,4,8,12,16,25$, and $32 \mu \mathrm{g})$. Rate of cell viability after treatment by TCE was determined using the MTT assay. After 24-72 $\mathrm{h}$ of incubation, $100 \mu \mathrm{l}$ of MTT reagent $(5 \mathrm{mg} / \mathrm{ml})$ solution was added to each well. Then, the plates were incubated for $4 \mathrm{~h}$ at $37^{\circ} \mathrm{C}$. After incubation, the MTT reagent and medium in each well were removed, and Sorenson buffer plus DMSO $(200 \mu \mathrm{l})$ were added to the wells and they were placed on a shaker incubator for $30 \mathrm{~min}$. Optical density (OD) of each well was measured by the enzyme-linked immunosorbent assay (ELISA) reader (Bio-Tek, USA) at $570 \mathrm{~nm}$. Moreover, $\mathrm{IC}_{50}$ level was determined by GraphPad Prism 6.0 software (GraphPad Software, USA).

\section{Scratch Test (Wound Healing Assay)}

In this method, MCF7, MDA231, and A549 cell lines were cultured on 6-well plates. Then, a sterile yellow pipette tip was used to create a scratch about $0.5 \mathrm{~mm}$ wide across the monolayer cell. After scratching, the detached cells were removed by washing with phosphate-buffered saline (PBS) solution and image was taken from the scratch region. Then, plates were treated with different concentrations of TCE and were incubated for $72 \mathrm{~h}$. Images of the wounded area were recorded using a light microscope at time intervals of $0,24,48$, and $72 \mathrm{~h}$. Finally, rate of cell migration in the wounded area was calculated by evaluating width of the scratch region.

\section{Extraction of Total RNA, cDNA Synthesis, and qRT-PCR}

The total RNA extraction was done from the treated and untreated cell lines $24 \mathrm{~h}$ after TCE treatment using an extraction kit according to the manufacturer's instructions (Takara Biotechnology Inc.). Takara cDNA synthesis kit (Takara, cat.No:RR037Q) was used for cDNA synthesis according to the manufacturer's protocol. So, $2 \mu \mathrm{g}$ of total extracted RNA was added to MMLV reverse transcriptase plus oligo-dT and random hexamer primers according to the manufacturer's protocol. The StepOne Real-time PCR System (Applied Biosystems, USA) and the SYBR Green gene expression Master mix (Takara, Korea) were applied to perform quantitative analysis. For this reason, $1 \mu \mathrm{M}$ of primer mix, $1.5 \mu \mathrm{l}$ of cDNA template, $7.5 \mu \mathrm{l}$ of nuclease-free water, and $10 \mu \mathrm{l}$ of SYBR green Premix were added to each micro tube. The glyceraldehyde-3phosphate dehydrogenase (GAPDH) gene was used as an endogenous control. The primers were designed by Oligo 7 software (Table 1). Relative expression of $m i R-21, m i R$ 34a, Caspase 9, Caspase 8, vimentin, Bcl2, Caspase 3, matrix metalloproteinase-13 (MMP-13), and Bax genes was determined by the $2(-\Delta \Delta \mathrm{Ct})$ method (Livak and Schmittgen, 2001).

\section{Statistical Analysis}

All the data were expressed as mean \pm standard deviation (SD). Statistical significance of variances between more than two samples were determined by analysis of variance (ANOVA) followed by Bonferroni's and Sidak's multiple comparisons and Unpaired Two-Sample Student's t-test (P-values $<0.05$ were significant). Statistical analysis was done via Graph Pad Prism 6 (Graph Pad Software, USA). 

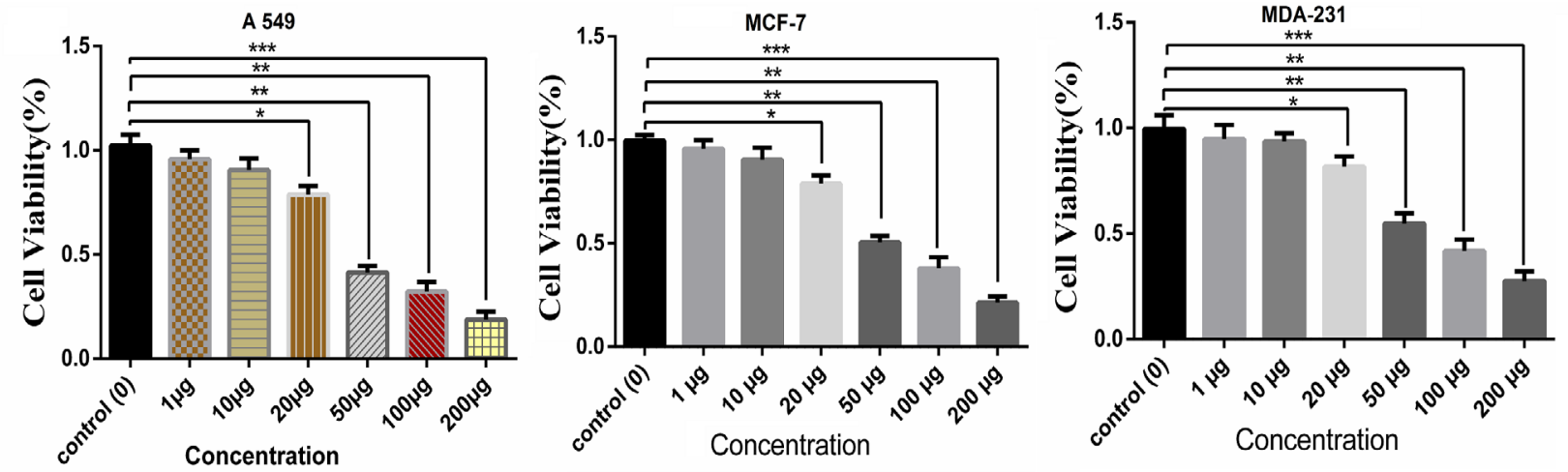

Figure 1. Effect of IC $\mathrm{IC}_{50}$ Concentration of TCE on A549, MCF-7, and MDA-231 Cell Proliferation: TCE decreased the cell survival to $17.6 \%, 21.4 \%$, and 27.21 in $200 \mu \mathrm{g}$ concentration compared to the control cells.

\section{Results}

Toxicity Effect of TCE on A549, MCF7, and MDA231 Cell Lines

For determining the cytotoxic effect of TCE on cancer cell viability, the A549, MCF7, and MDA231 cells were treated with different concentrations of TCE at time intervals of 24,48 , and $72 \mathrm{~h}$. Cell viability was considered by MTT assay in A549, MCF7, and MDA231cells, respectively. The results confirmed that the TCE decreased cell viability rate in a dose-dependent manner. Consequently, treating A549, MCF7, and MDA231 cell lines with $200 \mu \mathrm{g}$ of the TCE reduced survival rate by 17.6 ,
21.4 , and $27.21 \%$, respectively compared to the control untreated cell ( $\mathrm{p}<0.05$; Figure 1). Furthermore, an IC50 level was calculated by GraphPad Prism software 6.0 for A549 (42.32 $\mu \mathrm{g})$, MCF7 (50.21 $\mu \mathrm{g})$, and MDA-231 (53.87 $\mu \mathrm{g})$ cell lines.

Low Expression of MMP-13 and Bcl-2 and High Expression of miR-34a, Cas-9, Cas-3, and Bax in A549, MCF-7, and MDA-231 Cells after Treatment with TCE

Effects of the TCE on expression level of MMP-13, miR-21, Vimentin, Cas-8, Cas-3, miR-34a, Cas-9, Bax, and Bcl-2, in A549 (Figure 2), MCF-7 (Figure 3), and
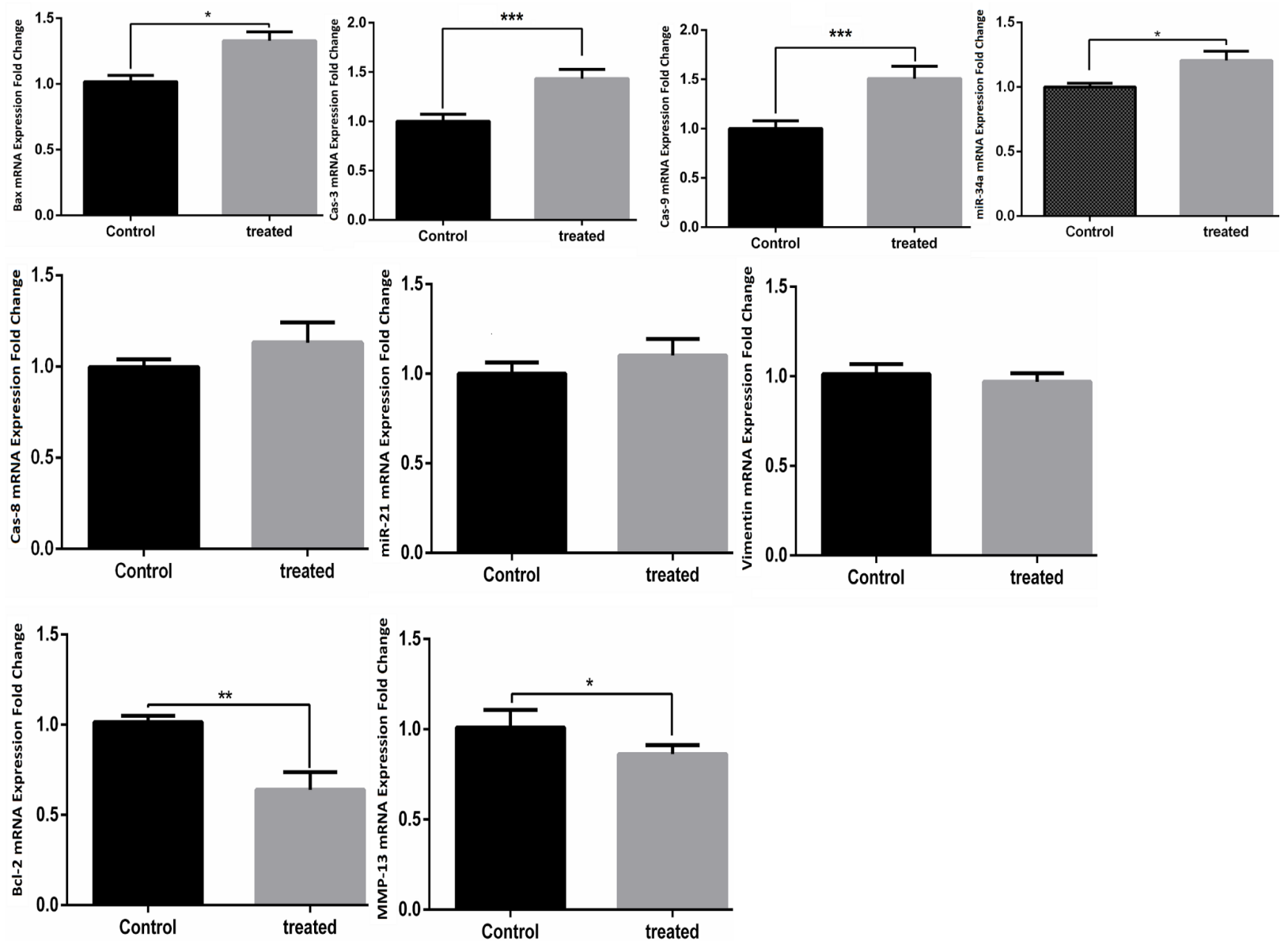

Figure 2. Effects of TCE Treatment on the Expression of BAX, Bcl-2, MMP-13, miR-21, miR-34a, Cas-3, Cas-8, Cas9 and Vimentin in A549 Cell Line. The data was presented as mean $\pm \mathrm{SD}(\mathrm{N}=3)(*=\mathrm{P}<0.05)$. 

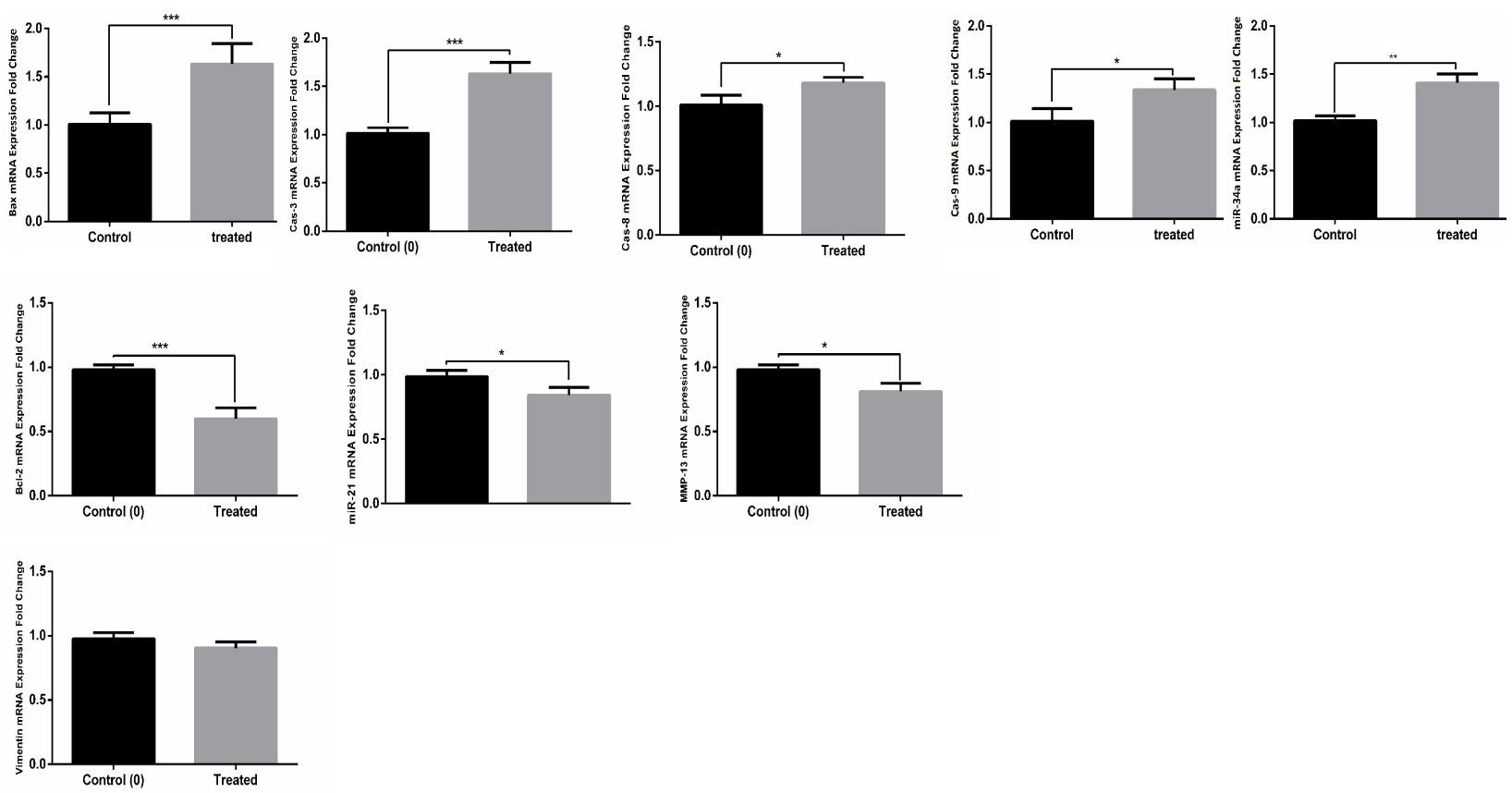

Figure 3. Effects of TCE Treatment on the Expression of BAX, Bcl-2, MMP-13, miR-21, miR-34a, Cas-3, Cas-8, Cas-9 and Vimentin in MCF-7 Cell Line. The data was presented as mean $\pm \mathrm{SD}(\mathrm{N}=3)(*=\mathrm{P}<0.05)$.

MDA-231(Fig. 4) cell lines were determined by real-time PCR. In the MCF-7 cells, $24 \mathrm{~h}$ after treatment with TCE, $m R N A$ expression level of the $m i R-21$ (p value: 0.016 , fold change: 0.237$)$, Bcl-2 ( $\mathrm{p}$ value: 0.00078 , fold change: 0.419 ) and MMP-13 (p value: 0.011 , fold change: 0.273 ), was reduced by $76.3,58.1$, and $72.7 \%$, respectively.

Also, mRNA level of miR-34a ( $\mathrm{p}$ valu:0.0059, fold change:1.73), Cas-8 (p value: 0.017 , fold change:1.21), Cas-3 (p value:0.00032 fold change:1.64), Cas-9 ( $p$ value:0.037, fold change:1.38), and Bax (p value:0.00043, fold change:1.61) was increased by $173,121,164,138$, and $161 \%$, respectively. There was no significant change in the $m R N A$ expression of Vimentin between control group and TCE-treated cells.

In the A549 cells, $24 \mathrm{~h}$ after treatment with TCE, mRNA level of MMP-13 (p value:0.041, fold change:0.218) (p value: 0.041 , fold change: 0.218 ) and Bcl-2 ( $\mathrm{p}$ value:0.0076, fold change:0.381) ( $\mathrm{p}$ value: 0.0076 , fold change: 0.381 ) was reduced by 78.2 and $61.9 \%$, respectively. Also, mRNA level of miR-34a (p value: 0.031 , fold change:1.21), Bax ( $\mathrm{p}$ value: 0.029 , fold change:1.36), Cas-3 (p value: 0.00058, fold change:1.47),
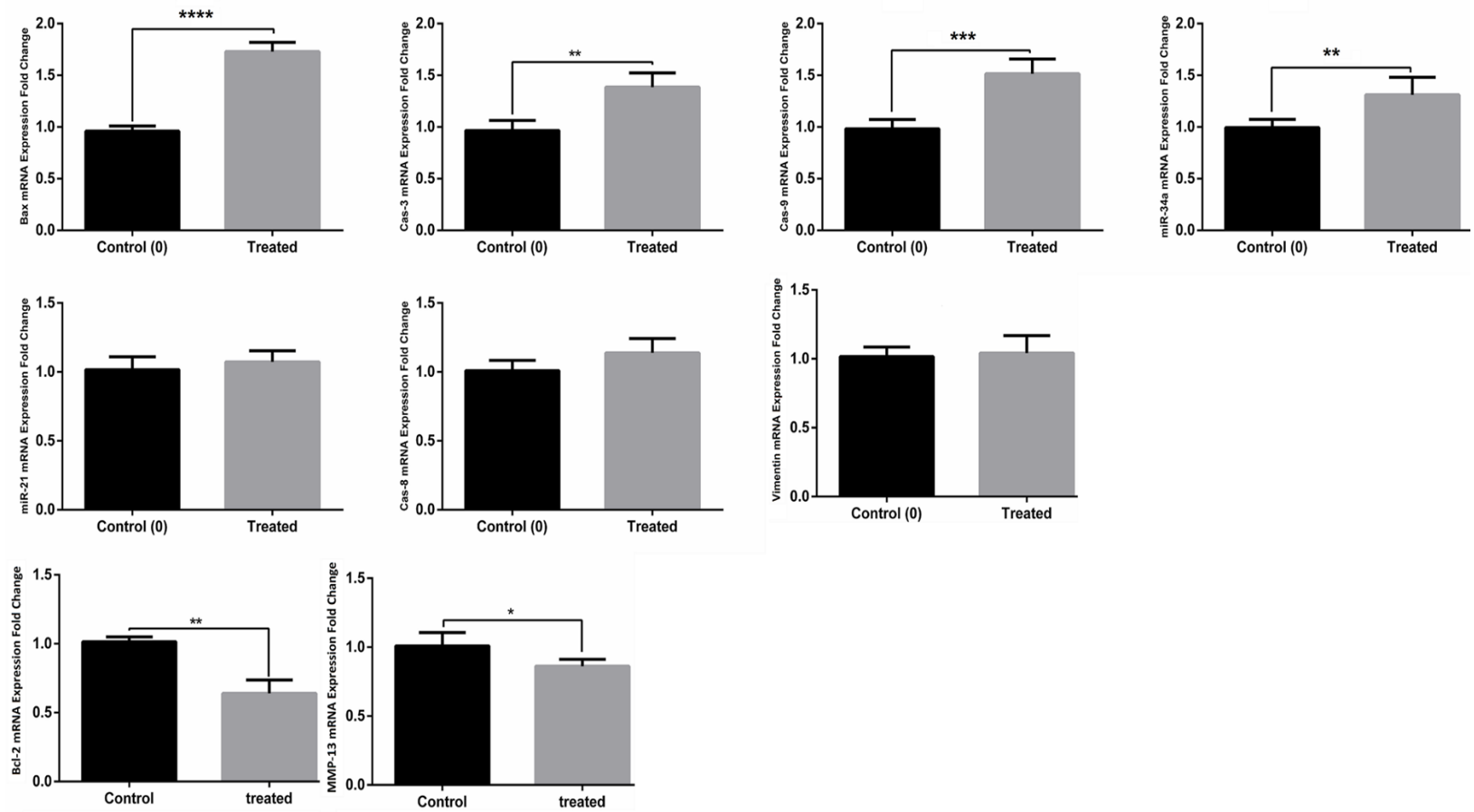

Figure 4. Effects of TCE Treatment on the Expression of BAX, Bcl-2, MMP-13, miR-21, miR-34a, Cas-3, Cas-8, Cas9 and Vimentin in MDA-231 Cell Line. The data was presented as mean $\pm \mathrm{SD}(\mathrm{N}=3)(*=\mathrm{P}<0.05)$. 
A549

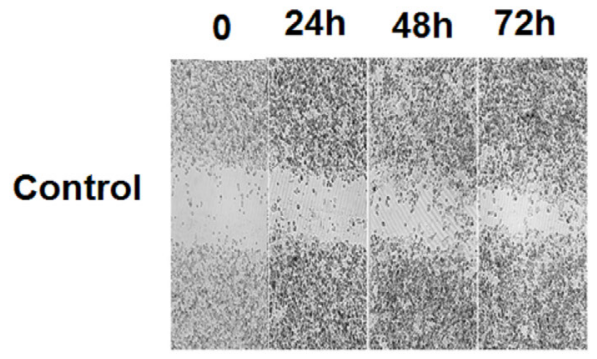

Treated

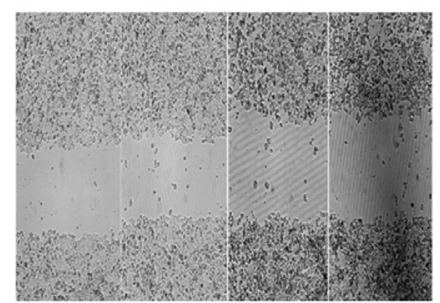

MCF-7
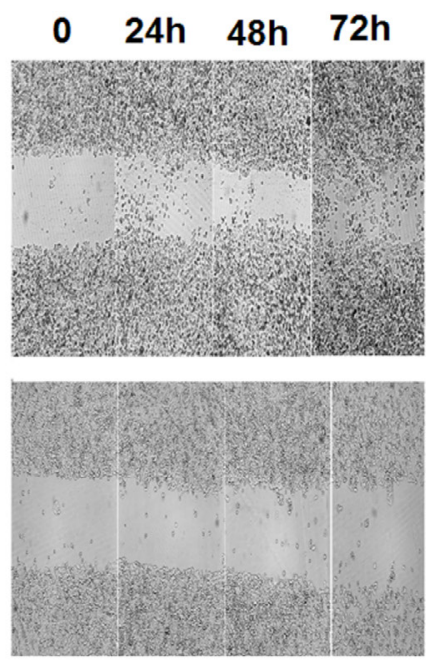

MDA-231

$24 \mathrm{~h} \quad 48 \mathrm{~h} \quad 72 \mathrm{~h}$
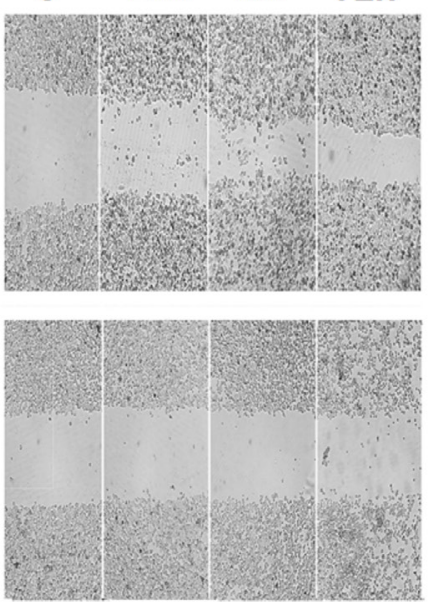

Figure 5. Results of Wound Healing Assay Shows that Untreated Cells (Control Group) have a Significant Further Migration in Comparison with Cells Treated with TCE.

Table 1. Primer Sequences Used for Real-Time Gene Expression Quantification of the Target Genes and miRNAs.

\begin{tabular}{|c|c|c|}
\hline Primer & $\begin{array}{l}\text { Forward } \\
\text { and reverse }\end{array}$ & Sequence \\
\hline \multirow[t]{2}{*}{ Bax } & Forward & 5'- GACTCCCCCCGAGAGGTCTT-3' \\
\hline & Reverse & 5'- ACAGGGCCTTGAGCACCAGTT -3' \\
\hline \multirow[t]{2}{*}{ Bcl-2 } & Forward & 5'- GAGCGTCAACCGGGAGATGTC -3' \\
\hline & Reverse & 5'- TGCCGGTTCAGGTACTCAGTC-3' \\
\hline \multirow[t]{2}{*}{ Caspase 3} & Forward & 5'-ATGGTTTGAGCCTGAGCAGA-3' \\
\hline & Reverse & 5'-GGCAGCATCATCCACACATAC-3' \\
\hline \multirow[t]{2}{*}{$\beta$-actin } & Forward & 5'-TCCCTGGAGAAGAGCTACG-3' \\
\hline & Reverse & 5'-GTAGTTTCGTGGATGCCACA-3' \\
\hline \multirow[t]{2}{*}{ Caspase 9} & Forward & 5'-GCAGGCTCTGGATCTCGGC-3' \\
\hline & Reverse & 5'-GCTGCTTGCCTGTTAGTTCGC-3' \\
\hline \multirow[t]{2}{*}{ Caspase 8} & Forward & 5'-ACCTTGTGTCTGAGCTGGTCT-3' \\
\hline & Reverse & 5'--GCCCACTGGTATTCCTCAGGC -3' \\
\hline $\operatorname{miR}-21$ & Exiqon & Product number:202,007 \\
\hline miR-34a & Exiqon & Product number: 202,860 \\
\hline \multirow[t]{2}{*}{ MMP-13 } & Forward & 5'-TGCAGAGCGCTACCTGAGATCATAC-3' \\
\hline & Reverse & 5'- GGAGCTTGCTGCATTCTCCTTCA-3' \\
\hline
\end{tabular}

and Cas-9 (p value:0.00023, fold change:1.49) was increased by $121,136,147$, and $149 \%$, respectively. There was no significant change in $m R N A$ expression level of miR-21, Vimentin, and Cas-8 between control group and TCE-treated cells.

In the MDA-231 cells, $24 \mathrm{~h}$ after treatment with TCE, mRNA expression level of MMP-13 ( $\mathrm{p}$ value:0.032, fold change:0.183) and Bcl-2 (p value:0.0023, fold change:0.439) was reduced by 81.7 and $56.1 \%$, respectively. In the same way, expression level of miR-34a (p value:0.0019, fold change:1.31), Cas-3 (p value:0.003, fold change:1.41), Cas-9 (p value:0.00041, fold change: 1.59 ), and $\operatorname{Bax}(\mathrm{p}$ value $<0.0001$, fold change:1.78) was increased by $131,141,159$, and $178 \%$ ,respectively. There was no significant alteration in mRNA expression level of Vimentin, miR-21, and Cas-8 between
TCE-treated cells and control group.

\section{TCE Prohibited Migration of A549, MDA-231, and MCF-} 7Cell Lines

For determining the effect of the TCE on migration ability of the A549, MCF-7, and MDA-231 cells, after treating cell lines with the TCE, it was found that the treated cells did not have an ability to migrate and cover the scratch region. But the control group had the ability to migrate and cover the scratch area. Briefly, the results of wound healing assay indicated a notable decrease in capability of the TCE-treated cells in migration to the scratch region (Figure 5).

\section{Discussion}

For the years, herbal medicine has played important roles in preventing and treating various ailments, as well as cancer and chronic diseases. In this regard, importance of innovative technologies as well as gene editing (Ahmadzadeh et al., 2019) and siRNA technologies (Shanehbandi et al., 2019; Zarredar et al., 2019) has not been able to reduce importance of herbal medicine as a source of therapeutic agents. Anti-cancer herbal drugs have received great attention to find harmless therapeutics with low toxicity. Suppressive effect of the TCE has been described on invasion, migration, and proliferation of various tumor cells including lung cancer ( $\mathrm{Chu}$ et al., 2007; Shanehbandi et al., 2019), hepatocellular carcinoma (Ko et al., 2003), and oral cancer (Yang et al., 2010). Thus, in the present study, it was hypothesized that treatment with the TCE may repress development, growth, and invasion ability of A549, MCF-7, and MDA-231 cell lines. Likewise, Yeh et al.,(2012) in their study confirmed the suppressive effect of the TCE on hepatocellular carcinoma (Yeh et al., 2012). Also, Lee et al., (2019) reported that the TCE had low cytotoxic effect on human HeLa and SiHa cervical cancer cells. Though, TCE has been found to inhibit mitogen-activated protein 
kinase (MAPK) and matrix metalloproteinase 9(MMP-9) pathway and decrease invasion and migration abilities of tumor cells (Lee et al., 2019). Also, Shanehbandi et al., (2020) found that the TCE promoted apoptosis, suppressed growth and migration rate in SW480 cell line by regulating expression of genes correlated with apoptosis and metastasis (Shanehbandi et al., 2019). In this research, the TCE displayed significant suppressive effects on proliferation, progression, and migration of A549, MCF-7, and MDA-231 cell lines. The final goal of the most therapeutic techniques for tumor treatment including radiotherapy and chemotherapy is promoting apoptosis and reducing cell development (Shekari et al., 2019). Apoptosis-related genes have essential role in numerous malignancies, particularly in cancer. In this study, after treating cancer cell lines by the TCE, Cas-9 expression was upregulated as the indicator of intrinsic apoptosis in all of the three cell lines Nevertheless, Cas- 8 as a mediator of extrinsic apoptosis induction did not display a significant expression in the $A 549$ and MDA-231 treated cells. But, Cas-8 displayed significant upregulation in the MCF-7 cell line. Also, in this study, after treatment with the TCE, Cas-3 which is related to mitochondrial and extrinsic apoptosis signaling pathways showed a significant upregulation. According to results of $q R T-P C R$, expression level of the $B c l-2$ was reduced in the treated cancer cells. On the other hand, Bax expression level was upregulated in the treated cells. Bakhshaiesh et al., (2015) indicated that $\mathrm{Bax} / \mathrm{Bcl} 2$ ratio might be a marker of susceptibility to apoptosis (Bakhshaiesh et al., 2015). Moreover, in this study MMP-13 as a vital member of the MMP family was meaningfully less expressed. Yang et al., (2010) demonstrated that the TCE could inhibit invasion and metastases of SCC-4 oral cancer by suppressing expression of urokinase plasminogen activator (u-PA), matrix metalloproteinase 2(MMP-2), and MMP-9 both in protein and mRNA levels (Yang et al., 2010). The remarkable role of MMP-13 in initiation of the extracellular MMP cascade has been proposed in the recent studies (Bakhshaiesh et al., 2015). Also, Chu et al., (2007) showed that the TCE had a dose-dependent suppressive effect on invasion ability and metastasis of Lewis lung carcinoma and A549 cells. Additionally, in the TCE-treated cell lines, expression level of $m i R-34 a$ (as a tumor suppressor) was upregulated (A549, MCF-7, and MDA-231). The miR-34a had suppressive effect on proliferation and invasion of tumor cells by activation of p53. Bcl-2 mRNA was also targeted by MiRNA-34a as an important factor in cancer cells (Chu et al., 2007). So, after treatment with the TCE, expression levels of $m i R-21$ and Vimentin (as a metastasis gene) were reduced. But, rate of change was not statistically significant. Therefore, according to the previous findings and results of the current study, the TCE might have ability to control malignancy and promote apoptosis in A549, MDA-231, and MCF-7cell lines.

In conclusion, our findings revealed that the TCE suppressed growth and induced apoptosis in A549, MCF-7, and MDA-231 cell lines. Also, the TCE reduced metastases and migration ability of cell lines by regulating expression level of genes correlated with metastasis and apoptosis. So, the TCE can be used as a candidate anti-cancer agent in treatment of lung and breast cancers.

\section{List of Abbreviations \\ $\mathrm{TC}=$ Terminalia Catappa \\ $\mathrm{TCE}=$ Terminalia Catappa Extract \\ $\mathrm{MMP}=$ Matrix metalloproteinase}

\section{Author Contribution Statement}

Habib Zarredar: Conceptualization, Visualization, Supervision. Amir Mahdi Khamaneh: Methodology, Software, Validation. Fatemeh Firozi Amoodizal: Software, Validation. Milad Asadi: Methodology, Software, Editing. Venus Zafari: Software, Validation. Ensiyeh Seyedrezazadeh: Software, Validation. Yeganeh Khalili: Software, Writing. Zahra Soleimani: Visualization, Writing. Hamed Sabagh Jadid: Visualization, Writing, Editing. Atefeh Ansarin: Visualization, Writing. Majid Khalili: Conceptualization, Methodology, Validation. Dariush Shanehbandi: Methodology, Supervision.

\section{Acknowledgements}

This study was supported by a grant from research deputy of Department of Tuberculosis and Lung Diseases Research Center, University Tabriz University of Medical Sciences, Tabriz, Iran.

\section{Ethics approval and consent}

This article does not contain any studies with human participants or animals performed by any of the authors. Also, this proposal has been reviewed and approved by the Ethics Committee of the Research center of the Tuberculosis and Lung Diseases Research Center at Tabriz University of Medical Sciences.

\section{Conflict of interest}

The authors declare that there is no conflict of interest associated with the manuscript.

\section{References}

Ahmadzadeh V, Farajnia S, Baghban R, et al (2019). CRISPRCas system: Toward a more efficient technology for genome editing and beyond. J Cell Biochem, 120, 16379-2.

Bakhshaiesh T, Armat M, Shanehbandi D, et al (2015). Arsenic trioxide promotes paclitaxel cytotoxicity in resistant breast cancer cells. Asian Pac J Cancer Prev, 16, 5191-7.

Bertucci F, Finetti P, Birnbaum D (2012). Basal breast cancer: a complex and deadly molecular subtype. Curr Mol Med, 12, 96-110.

Bray F, Jemal A, Grey N, Ferlay J, Forman D (2012). Global cancer transitions according to the Human Development Index (2008-2030): a population-based study. Lancet Oncol, 13, 790-1.

Chen P-S, Li J (2006). Chemopreventive effect of punicalagin, a novel tannin component isolated from Terminalia catappa, on H-ras-transformed NIH3T3 cells. Toxicol Lett, 163, 44-3.

Chen P-S, Li J, Liu T, Lin T (2000). Folk medicine Terminalia catappa and its major tannin component, punicalagin, are effective against bleomycin-induced genotoxicity in Chinese hamster ovary cells. Cancer Lett, 152, 115-2. 
Chu S, Yang S, Liu S, et al (2007). In vitro and in vivo antimetastatic effects of Terminalia catappa L. leaves on lung cancer cells. Food Chem Toxicol, 45, 1194-201.

Ezeokonkwo C, Dodson C (2004). The potential of Terminalia catappa (tropical almond) seed as a source of dietary protein. J Food Qual, 27, 207-9.

Fan Y, Xu L, Gao J, et al (2004). Phytochemical and antiinflammatory studies on Terminalia catappa. Fitoterapia, 75, 253.

Ferlay J, Soerjomataram I, Dikshit R, et al (2015). Cancer incidence and mortality worldwide: sources, methods and major patterns in GLOBOCAN 2012. Int J Cancer, 136 359-6.

Ferlay J, Shin H, Bray F, et al (2010). Estimates of worldwide burden of cancer in 2008): GLOBOCAN 2008. Int J Cancer, 127, 2893-7.

Fidler IJ (1989). Origin and biology of cancer metastasis. Cytometry, 10, 673-80.

Kinoshita S, Inouse Y, Nakama S, Ichiba T, Aniya Y (2007). Antioxidant and hepatoprotective actions of medicinal herb, Terminalia catappa L. from Okinawa Island and its tannin corilagin. Phytomedicine, 14, 755-2.

Ko T, Weng Y, Lin S, Chiou R (2003). Antimutagenicity of supercritical $\mathrm{CO} 2$ extracts of Terminalia catappa leaves and cytotoxicity of the extracts to human hepatoma cells. J Agric Food Chem, 51, 3564-7.

Lee C, Yang S, Wang P, et al (2019). Antimetastatic effects of Terminalia catappa leaf extracts on cervical cancer through the inhibition of matrix metalloprotein-9 and MAPK pathway. Environ Toxicol, 34, 60-6.

Li R, Sonik A, Stindl R, Rasnick D, Duesberg P (2000). Aneuploidy vs. gene mutation hypothesis of cancer: recent study claims mutation but is found to support aneuploidy. PNAS, 97, 3236-1.

Rusciano D, Burger M (1992). Why do cancer cells metastasize into particular organs?. Bioessays, 14, 185-94.

Saika K, Sobue T (2013). Cancer statistics in the world. Cancer Chemotherapy, 40, 2475-0.

Shanehbandi D, Zarredar H, Asadi M, et al (2019). Anticancer impacts of Terminalia catappa extract on SW480 colorectal neoplasm cell line. $J$ Gastrointest Cancer, 2019, 1-7.

Shekari N, Asghari F, Haghnavaz N, et al (2019). Let-7a could serve as a biomarker for chemo-responsiveness to docetaxel in gastric cancer. Anticancer Agents Med Chem, 19, 304-9.

Stewart B, Wild C (2015). World cancer report 2014, WHO.

Tan W, Lu J, Huang M, et al (2011). Anti-cancer natural products isolated from chinese medicinal herbs. Chin Med, 6, 27.

Yang S, Chen M, Hsieh Y, et al (2010). Antimetastatic effects of Terminalia catappa L. on oral cancer via a down-regulation of metastasis-associated proteases. Food Chem Toxicol, 48, 1052-8.

Yeh C, Hsieh M, Hsieh Y, et al (2012). Corrigendum to Terminalia catappa Exerts Antimetastatic Effects on Hepatocellular Carcinoma through Transcriptional Inhibition of Matrix Metalloproteinase- 9 by Modulating NF-B and AP-1 Activity. Evid Based Complement Alternat Med, 2012, 595292.

Zarredar H, Farajnia S, Ansarin K, et al (2019). Synergistic effect of novel EGFR inhibitor AZD8931 and p38 $\alpha$ siRNA in lung adenocarcinoma cancer cells. Anticancer Agents Med Chem, 19, 638-4.

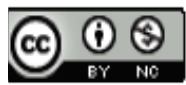

This work is licensed under a Creative Commons AttributionNon Commercial 4.0 International License. 